\title{
Successful Pelvic Exenteration for T4b Rectal Cancer: A Case Report
}

\section{GZ Bounab ${ }^{1}$, S Bicha ${ }^{2}$, H Rahmoune ${ }^{3 *}$, N Boutrid ${ }^{3}$, R Sayoud ${ }^{1}$, H Boutrid $^{4}$ and N Lemdaoui ${ }^{2}$}

${ }^{1}$ Urology Department, EHS Daksi, Constantine, Algeria

${ }^{2}$ General Surgery "B" Department, Ben Badis University Hospital of Constantine, Algeria

${ }^{3}$ LMCVGN Research Laboratory, Setif 1 University, Algeria

${ }^{4}$ Department of Obstetrics-Gynecology, University Hospital of Babeloued, Algiers-1

University, Algeria

*Corresponding Author: H Rahmoune, LMCVGN Research Laboratory, Setif 1

University, Algeria.

DOI: $10.31080 /$ ASGIS.2020.03.0159

\section{Abstract}

Pelvic exenteration (PE) or pelvectomy is defined as radical "En Bloc" resection of two or more contiguous pelvic organs, followed by reconstruction or diversion of visceral functions.

PE was first reported by Brunschwig in 1948 as a particular and radical surgery for advanced and recurrent cancer and considered as "the most radical surgical attack so far described for pelvic cancer". Its broad indications are curative strategies discussed by a multidisciplinary team; PE must be done "En Bloc" with negative margin status.

We report a case of a rectal mucinous carcinoma in a 38 year-old man. The patient benefited radical treatment through total PE with terminal colostomy and urinary diversion (ureterostomy by Bricker's method) with good post-operative outcomes.

Keywords: Pelvic Exenteration; Operative Surgical Procedures; Rectal Neoplasms; Pelvic Neoplasms; Urinary Diversion

\section{Introduction}

PE was first reported by Brunschwig in 1948 as a particular and radical surgery for advanced and recurrent cancer and considered as "the most radical surgical attack so far described for pelvic cancer" [1].

Pelvic exenteration (PE) or pelvectomy is defined as radical En Bloc resection of two or more contiguous pelvic organs, followed by reconstruction or diversion of visceral functions, with or without resecting the perineum [2].

The Mesh (Medical Subject Headings, National Library of Medicine, USA) definition of PE is even wider: "Removal of all of the organs and adjacent structures of the pelvis. It is usually performed to surgically remove cancer involving the bladder, uterine cervix, or rectum" [3].
It is indicated as a curative treatment of locally advanced pelvic floor cancers in the aim of having a monoblock resection with negative margin status (R0).

These procedures are onerous, requiring a precise and exhaustive preoperative assessment, a well-codified preparation and usually reserved for selected patients without extra-pelvic disease in good general health. It is an operative technique using urological, gynecological, digestive and plastic techniques, along with a perfect knowledge of the highly complex anatomy of the pelvis.

PE increases overall survival in patients treated with curative intent, although it is associated with lengthy operating times (5 $14 \mathrm{~h}$ ), substantial blood loss, and high perioperative morbidity (40 - 90\%) and mortality rate (0 - 24\%) [4,5]. 


\section{Case Report}

We report the case of a 38-year-old male patient, without previous personal nor familial history, presenting to surgical emergencies for acute hematochezia.

At clinical examination, the patient has no other functional nor physical signs. Digital rectal examination completed by rectoscopy found an ulcerative-budding process of the lower rectum. A biopsy assessed the histological type as a mucinous colloid carcinoma.

Locoregional and distant extension assessment included a Pelvic MRI describing an involvement of the sphincteric complex, the seminal vesicles and the fascia recti (Figure 1). The thorax-abdomen-pelvis CT did not depict additional finding.

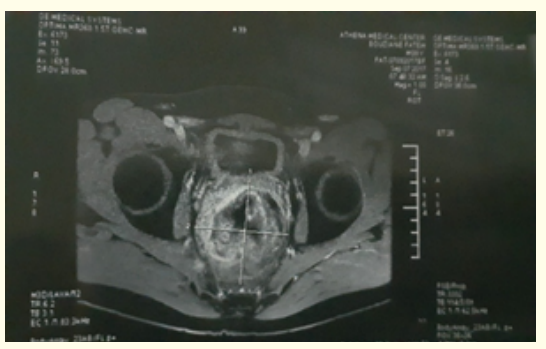

Figure 1: Rectal tumor with local infiltration.

The tumor was classified as T4bN1aM0.

After multidisciplinary team meeting, a complete resection procedure was decided: an initial colostomy (to protect the patient from acute complications during the surgeries), followed by concomitant neoadjuvant radio-chemotherapy (25 sessions at a dose of 54 gray), and finally a pelvic exenteration after 8 weeks (second MRI: T4bN1aM0).

The PE was realized in three principal times:

- Abdominal time: Vascular control, release of the rectum, lymph nodes dissection, colostomy terminalization and finally, total cysto-prostatectomy.

- Perineal time: Release of the rectum and the anal canal, enbloc exteriorization of the tumor.
- $\quad$ Reconstitution time of the urinary system (urinary diversion) by a double trans-ileal ureterostomy according to Bricker's method.

At the end, a drainage at the Douglas pouch and facing the Bricker diversion site was installed.

Postoperative period was free from complications and drains were removed at day 10 . The patient was discharged at day 13 .

The pathology results of the surgically extracted piece was good: negative margins with $\mathrm{R} 0$ resection $(\mathrm{T} 4 \mathrm{bN} 1 \mathrm{~b})$.

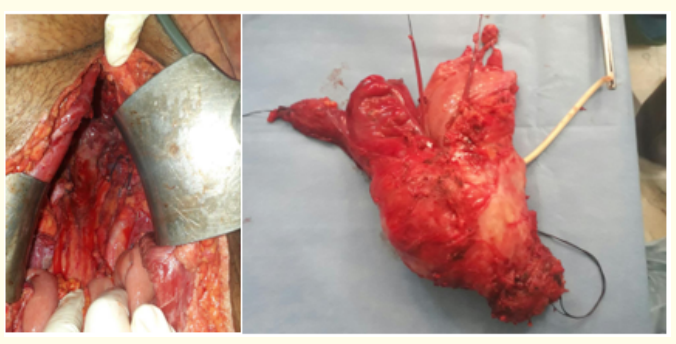

Figure 2: Left: Per-operative time, Right: Resected piece.

\section{Discussion}

PE is an operative technique using urological, gynecological, digestive and plastic techniques, along with a perfect knowledge of the highly complex anatomy of the pelvis.

A pre-, per- and post operative management of PE is mandatory and requires:

- $\quad$ A good indication; therefore, careful patient selection balancing risks and benefits is a crucial step when discussing this high-risk, high-return surgery, particularly locally advanced tumors T3, T4 as well as tumor recurrences, and serious sequelae of radiotherapy. The best indication, which represents only $25 \%$ of cases [6], is the isolated Centro-pelvic recurrence not attached to the pelvic walls.

- A preoperative assessment of total resection possibility (pelvic MRI) and remote extension (PET Scan). 
- A selection of patients based on both general and oncological criteria. Particularly, a poor nutritional status is associated with an increased risk of complication including anastomotic leak [7]. Optimization of nutritional status, if possible, has thus been recommended and has demonstrated improved outcomes following other major abdominal and oncologic procedures [7].

Operational difficulties are various, such as venous bleeding, the difficulty to get clear margins if local recurrence and to avoiding contamination of the operating field by tumor cells, as well as the difficulty of having a good dissection plan especially for irradiated patient.

The PE technique itself involves three major steps:

1. Exeresis; whether partial or total.

2. Diversion techniques for organ functions: Reconstruction of the digestive, urinary and gynecological system.

3. Procedure for treating the pelvic-perineal cavity.

The five-year recurrence-free survival after PE is estimated at 25 to $40 \%$ [8]. The histological (cancer) type and the primary tumor location are important prognostic factors as well.

Locally advanced primary rectal cancer has a better prognosis with five-year overall survival (5yS) of 55 to $65 \%[8,9]$.

The oncological outcome remains a priority via a large en-bloc resection to get negative margins that improve overall postoperative survival. In fact, negative margin status and perioperative radiation or chemoradiation have a dramatic impact on the survival rate after PE (estimated at $32-47 \%$ for overall survival and 40 - 52\% for recurrence-free survival) [10].

Thus, PE is an important surgical procedure as a curative strategy in locally invasive rectal neoplasms.

\section{Conclusion}

Pelvic exenteration for locally invasive cancer needs a deep knowledge of cancer surgical techniques, carried out by multidisciplinary, experienced teams.

The oncological objective remains a top-priority and requires a monoblock resection.
Negative margin status and perioperative radiation or chemoradiation are associated with decreased risk of death.

\section{Acknowledgment}

The authors would thank the Pathology, Oncology and Radiotherapy departments (Ben Badis University Hospital of Constantine) for their kind support.

\section{Support}

The authors (H Rahmoune, N Boutrid, N Lemdaoui) are supported by grants from the Directorate General for Scientific Research and Technological Development (DGRSDT), MESRS, Algeria.

\section{Bibliography}

1. Brunschwig A. "Complete excision of pelvic viscera for advanced carcinoma. A one-stage abdominoperineal operation with end colostomy and bilateral ureteral implantation into the colon above the colostomy". Cancer 1.2 (1948): 177-183.

2. Chatterjee S., et al. "National trends in total pelvic exenteration for gynecologic malignancies". American Journal of Obstetrics and Gynecology 215.3 (2016): 395-396.

3. Pelvic Exenteration.

4. Brown KG., et al. "Pelvic exenteration surgery: the evolution of radical surgical techniques for advanced and recurrent pelvic malignancy". Diseases of the Colon and Rectum 60.7 (2017): 745-754.

5. Quyn AJ., et al. "Palliative pelvic exenteration: clinical outcomes and quality of life". Diseases of the Colon and Rectum 59.11 (2016): 1005-1010.

6. Zhao J., et al. "Patterns and prognosis of locally recurrent rectal cancer following multidisciplinary treatment". World Journal of Gastroenterology: WJG 18.47 (2012): 7015-7020.

7. Sun Z., et al. "Nutritional risk screening 2002 as a predictor of postoperative outcomes in patients undergoing abdominal surgery: a systematic review and meta-analysis of prospective cohort studies". PloS One 10.7 (2015): e0132857.

8. Bozzetti F., et al. "Postoperative complications in gastrointestinal cancer patients: the joint role of the nutritional status and the nutritional support". Clinical Nutrition 26.6 (2007): 698-709. 
9. Yang TX., et al. "Pelvic exenteration for rectal cancer: a systematic review". Diseases of the Colon and Rectum 56.4 (2013):

519-531.

10. Konstantinidis IT., et al. "National postoperative and oncologic outcomes after pelvic exenteration for T4b rectal cancer". Journal of Surgical Oncology (2020).

\section{Assets from publication with us}

- Prompt Acknowledgement after receiving the article

- Thorough Double blinded peer review

- Rapid Publication

- Issue of Publication Certificate

- High visibility of your Published work

Website: www.actascientific.com

Submit Article: www.actascientific.com/submission.php Email us: editor@actascientific.com

Contact us: +919182824667 